\title{
A hypertext-based tutorial with links to the Web for teaching statistics and research methods
}

\author{
CHRISTOPHER KOCH and JOETTA GOBELL \\ George Fox University, Newberg, Oregon
}

\begin{abstract}
An online tutorial for research design and statistics is described. This tutorial provides a way for students to learn how scales of measure, research design, statistics, and graphing data are related. The tutorial also helps students determine what statistical analysis is appropriate for a given design and how the results of the analysis should be plotted in order to effectively communicate the results of a study. Initial research suggests that students using the tutorial are more accurate in their decisions about the design and statistics associated with a study. Students are also more confident in the decisions and find them easier to make when using the tutorial. Furthermore, practice with the tutorial appears to improve problem-solving ability in subsequent design and statistics scenarios. Implications for teaching statistics and research design are discussed.
\end{abstract}

This paper presents a tutorial ${ }^{l}$ available on the WorldWide Web (WWW) that consists of a series of decision paths concerning statistics and research design, along with pertinent definitions, explanations, figures, and links to additional statistics and research method sites. The tutorial can be used to supplement either statistics or research method texts. Statistics texts are written to present statistical procedures, and research method texts are written to address the pros and cons of particular research designs; therefore, this tutorial also serves the purpose of showing the relationship between the two.

The beginning chapters of introductory statistics texts typically cover the scientific method, measurement scales, graphing data, and descriptive statistics. As researchers, we measure empirical observations in some way. Therefore, the significance of the scales of measure is often clearly articulated in statistics texts (e.g., Velleman \& Wilkinson, 1993). Different scales have different characteristics and limitations that may limit the types of statistical analyses that can be conducted (see, e.g., Pedhazur \& Schmelkin, 1991). An understanding of scales of measure enables students to be aware of these limitations and encourages them to focus on how they want to measure a particular phenomenon. Unfortunately, the foundation of statistical analysis described in the early chapters of texts is often lost in later chapters, as students become increasingly concerned with understanding how to conduct a particular statistical test and less concerned with the theoretical underpinnings of analysis. Furthermore, the relevance of graphing, another topic covered in the beginning chapters of most statistics texts, seems to fade as students concentrate on interpreting results from parametric and nonparametric tests (see, e.g., Loftus, 1993,

Correspondence concerning this article should be addressed to $\mathrm{C}$. Koch, Department of Psychology, George Fox University, 414 N. Meridian St., Newberg, OR $97132-2697$ (e-mail: ckoch a georgefox.edu).
1996). In addition, the material on graphing data is typically rudimentary and limited to histograms, stem-andleaf displays, and bar charts. Authors usually do not revisit graphing when describing parametric and nonparametric tools. Therefore, students are often confused on how to present their results in graphical form (see, e.g., Estes, 1997).

Statistics and research design are often integrated in research method courses by statistically analyzing research projects (see, e.g., Dunn, 1996). However, this practice may limit students' understanding to the design and analysis appropriate for a particular project. Gathering data to analyze throughout a statistics class has also been suggested as a way to demonstrate the relationship between design and statistics (Thompson, 1994; also Lutsky, 1986). Unfortunately, this approach constrains learning to survey methods and allows for minimal experience with experimental and correlational research (Bear, 1995). Finally, although some authors incorporate statistics throughout a research method text (e.g., Shaughnessy \& Zechmeister, 1997), statistics are typically addressed in an appendix.

\section{Decision Charts for Research Design and Statistics}

Koch and Gobell (1999) have developed a series of decision charts to help students integrate statistics and research design. The first chart focuses on appropriate descriptive statistics. Depending on which scale of measure the study involves, students discover the best measure of central tendency and variability, along with the most appropriate way to graph the data (Figure 1). A second decision chart is used to determine the appropriate statistics to use when examining the relationship between two variables or when examining differences between groups of categorical data (Figure 2). Finally, the last decision chart helps determine the design of a study and the statistical tests appropriate for that design. Again, the best method for graphing the results is also presented (Figure 3 ). Thus, 


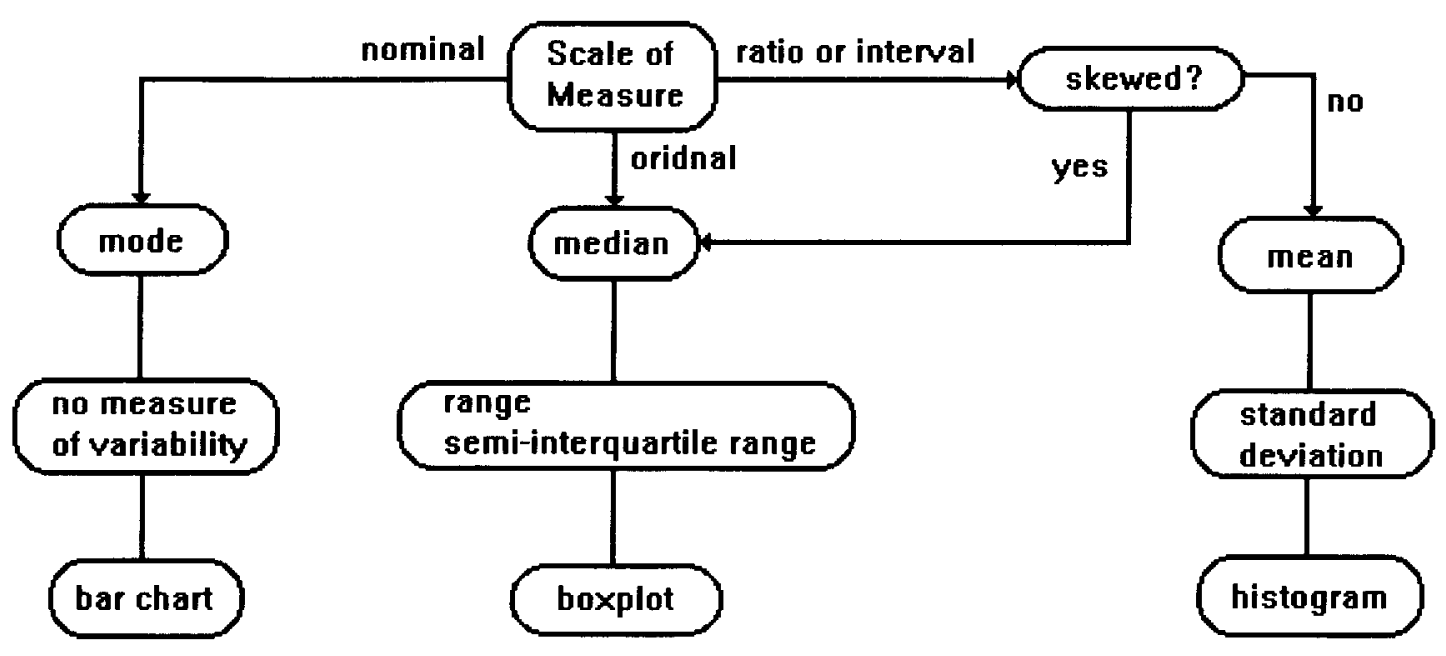

Figure 1. Decision chart design by Koch and Gobell (1999) for descriptive statistics.

the decision charts can be used with descriptions of particular research situations to help students understand how various factors determine the research design, statistics, and graphs on a study-by-study basis. A second benefit of these decision charts is that they combine statistics, research design, and graphing into one process, thereby preventing the separation of these topics in the student's learning experience.

Unfortunately, the functionality of the decision charts is limited by the paper medium in which they are presented. Space confines how much information the charts can contain and how readable the charts will be. Further-

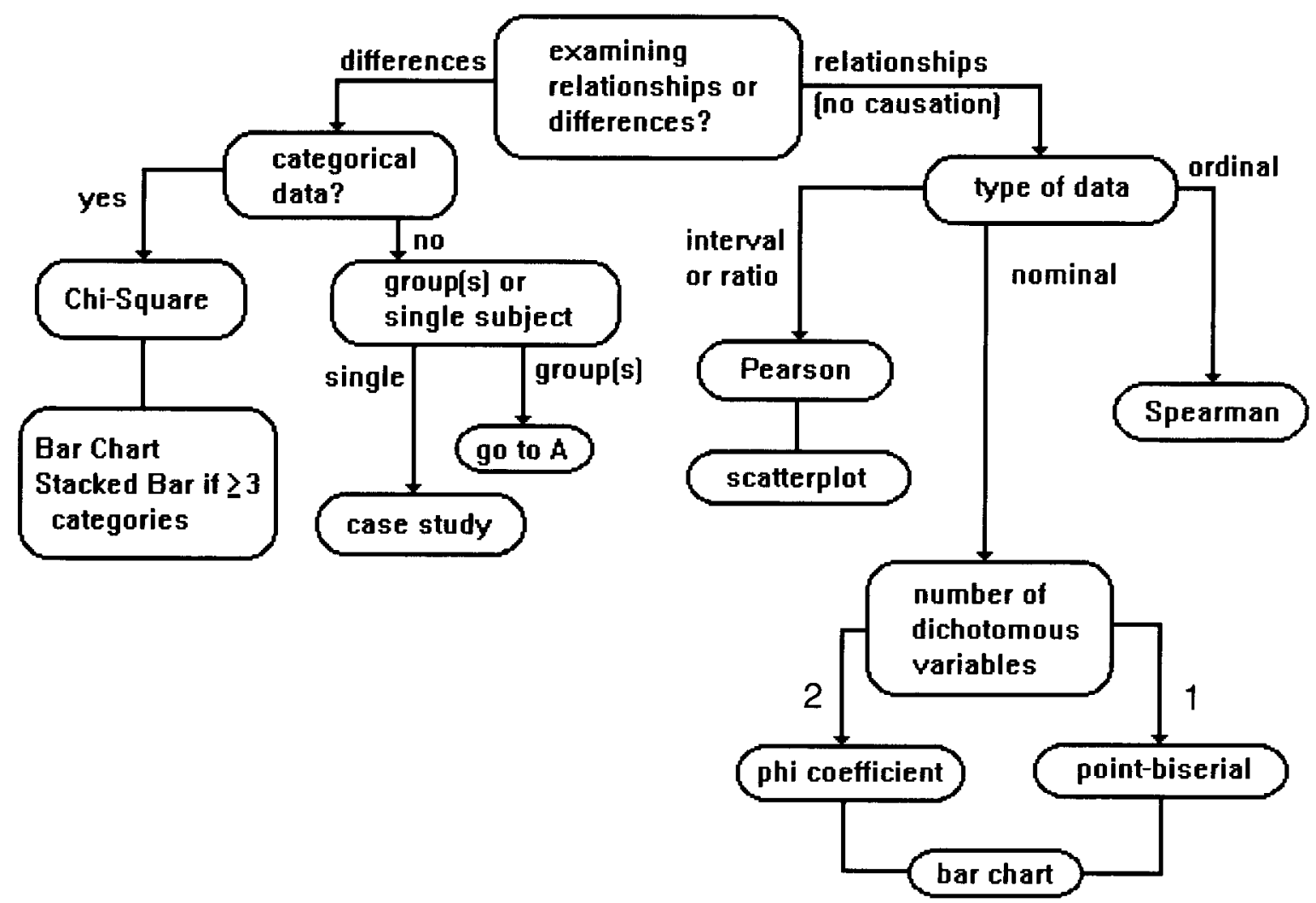

Figure 2. Decision chart design by Koch and Gobell (1999) for distinguishing between correlational and experimental studies. 


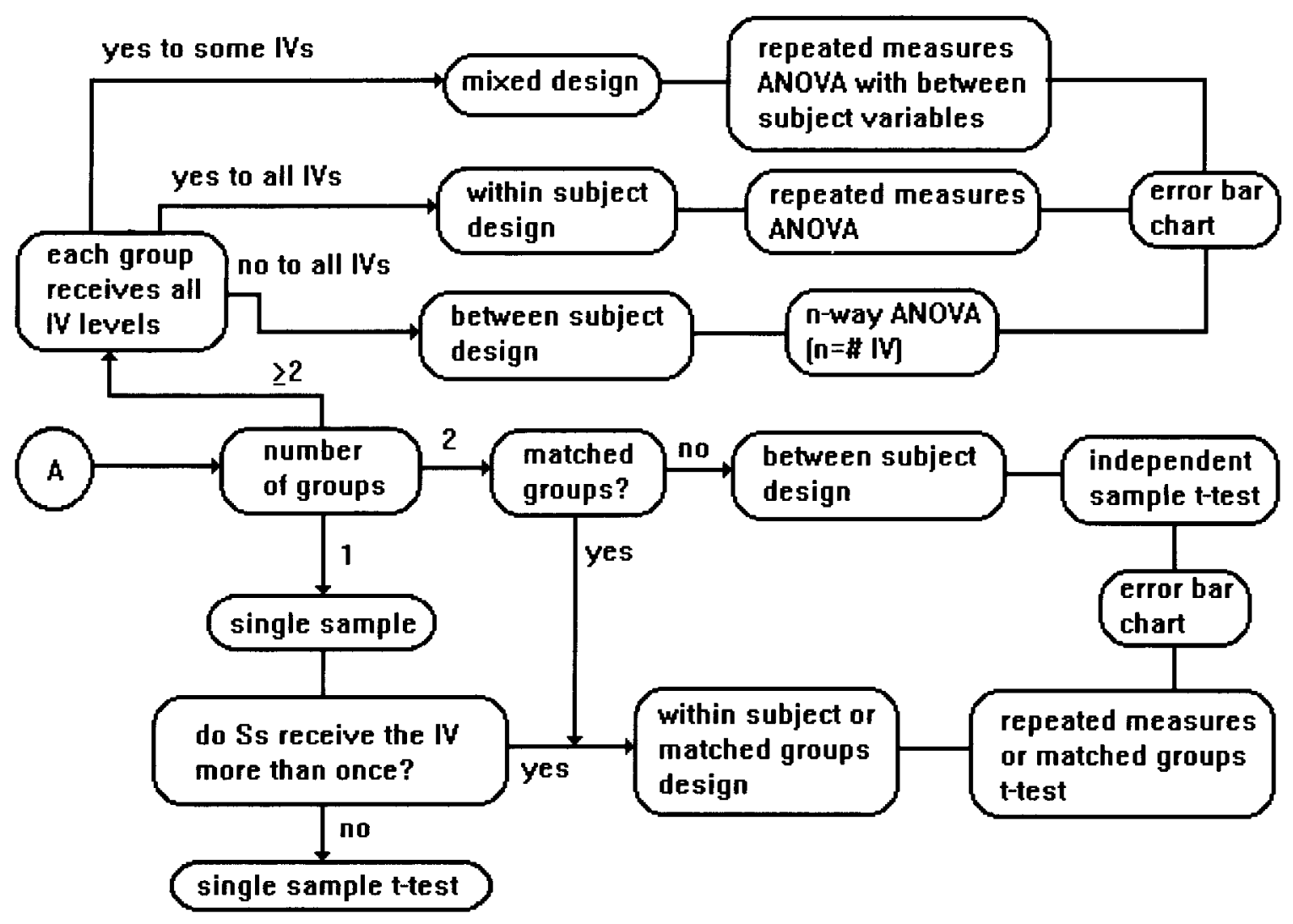

Figure 3. Decision chart design by Koch and Gobell (1999) for determining the appropriate inferential statistics and how to graph the results.

more, space also restricts the use of additional explanations and definitions for making the decision process easier as students progress through the charts. Therefore, we sought a presentation medium that provides greater flexibility in designing the decision charts. Hypertext markup language (HTML) and the WWW provide the flexibility needed to make the decision charts both more comprehensive and interactive.

\section{Benefits of Online Tutorials}

First, the WWW provides an easily accessible medium for presenting the decision charts. A Web site also provides an efficient method for making enhancements to the decision charts available. Second, HTML allows users to link from place to place without regard to the underlying structure of the decision charts. Therefore, the decision charts can become quite complex, but the user will only need to navigate through a simple hypertextlinked tutorial instead of a difficult-to-follow decision tree presented on paper. In addition, a variety of enhancements to the decision charts can be easily incorporated into HTML.

Enhancements can include adding definitions, providing examples of particular designs and statistics, providing examples of the various ways data can be graphed, and showing how to use particular statistics packages.
Enhancements can also involve linking to any of the numerous related resources available on the WWW. For instance, Cozby (1997b) provides links to WWW sites that are related to each chapter in his research methods text (Cozby, 1997a). Similarly, Trochim (1997a) provides links on the WWW to other web sites on social research and research methods, research method tutorials, and an online textbook on applied research methods. Trochim (1997b) also provides a series of 35 tutorials related to research methods. Because the tutorials were written by students for students, they are very readable and easy to understand. However, the tutorials are also fairly extensive. Tutorial topics range from avoiding threats to internal validity to multitrait-multimethod matrices. Each tutorial contains explanatory text, followed by a question that the student must answer. Depending on the answer, the student is directed to an appropriate path within the tutorial. Thus, these links can help explain terms and concepts specific to research methods. On-line statistics textbooks can similarly be used to help explain various statistical terms and concepts related to research design (de Leeuw, 1997; Lane, 1997). Therefore, enhancements to the decision charts are not limited to adding a path to another statistic, but enhancements can also provide access to additional information that makes learning statistics and research methods easier. 
Finally, the potential volume of student users on the WWW and the ease with which electronic feedback can be transmitted allow students to provide valuable information concerning the utility of an on-line tutorial. This information can then be used to tailor the tutorial to the needs of the students in order to facilitate learning.

\section{DESIGN OF ON-LINE TUTORIAL}

The Design -Statistics Finder tutorial (Koch \& Gobell, 1997) is structured after the decision charts developed by Koch and Gobell (1999). However, the on-line tutorial benefits from the flexibility of the WWW, as discussed above. Like the paper version of the decision charts, stu- dents answer a series of questions about the variables they are using. The responses lead the students to the type of design they have, along with the appropriate inferential statistics to conduct and instruction on how to graph the results. Unlike the paper version, however, the on-line tutorial provides definitions, links to related sites, and pictures of how to enter and analyze data with SPSS.

A simple example illustrates the type of information students gain by using the tutorial. Suppose we are interested in examining the usefulness of a particular teaching tool (e.g., the online tutorial). Furthermore, suppose that we randomly assign students into two groups. One group uses the teaching tool, whereas the second group does not. We then compare how well the two groups per-

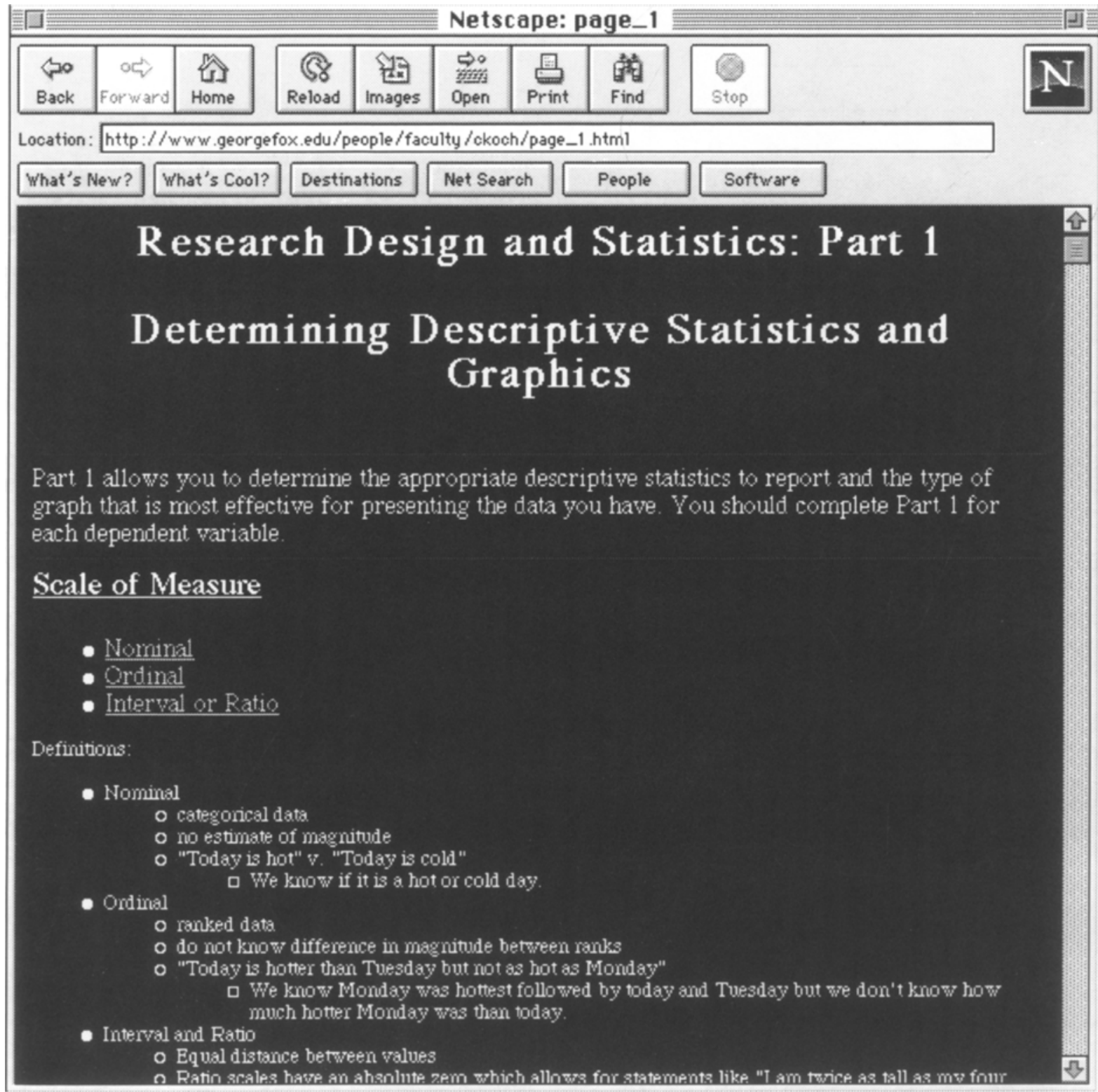

Figure 4. First screen of the on-line tutorial, which addresses that scale of measure used with the dependent variable. 
form on a quiz. The first screen of the tutorial addresses the scale of measure (Figure 4). Definitions and examples of each scale are provided. Selecting interval scale leads the student to a screen dealing with the shape of the distribution of scores. For this example, we can assume that the distribution is normal. The next screen in the tutorial displays the descriptive statistics (i.e., the mean and standard deviation) to use and how to graphically present the data (i.e., a histogram). Once the descriptive statistics are determined, students can then search for the appropriate statistical test. First, students must determine whether they are examining relationships or differences. In this case, we are interested in the difference between the group using the new teaching tool and the group not using it. There is then a series of screens asking for information concerning the type of data being collected and the nature of the groups (e.g., the number of groups, whether or not they are matched, if measures are repeated). In our example, the students are randomly assigned to two groups. Therefore, we find that we have a betweensubjects design and should use an independent samples $t$ test to analyze the data. Finally, the tutorial recommends that any differences between the two groups be displayed, using an error bar chart.

\section{EVALUATING THE DESIGN-STATISTICS TUTORIAL}

In order to examine the benefits of the Design-Statistics Finder, two simple studies were conducted, comparing the performance of two groups on research problems. In the first study, students from two sections of a research methods course were randomly assigned to two groups. In solving the first problem, one group $(n=12)$ used the Design-Statistics Finder, whereas the other group ( $n=$ 14) solved the problem without assistance. However, the group using the Design-Statistics Finder to solve the first problem solved the second problem without assistance. The group who solved the first problem without assistance used Design-Statistics Finder to solve the second problem. This procedure was employed to examine order effects. Both problems were modified versions of experimental dilemmas presented in Elmes (1995). The problems were matched for difficulty by including similar types of information (e.g., information about the variables) and requiring similar tests (i.e., an independent sample $t$ test and a repeated measure analysis of variance [ANOVA]).

The students were instructed to determine the type of design used, which descriptive and inferential statistics to use, and how to graph the data for each problem. Rosnow and Rosnow (1992) suggest that readers of psychological research should be able to determine an appropriate research design after reading the background information to the study and should also be able to determine what statistics are appropriate before they read the results section of a paper. Therefore, this task is similar to what students should do as consumers of psycho- logical research. After completing each problem, the students rated how confident they were in choosing the design, descriptive and inferential statistics, and graph, using a 5-point scale, with 1 meaning not at all confident and 5 meaning very confident. They similarly rated how easy it was to determine the design, statistics, and graph.

\section{Accuracy of Problem Solving}

Correct answers were assigned one point, whereas incorrect responses received no credit. A total accuracy score was calculated by adding the number of points received. Total scores could range from zero (no correct answers) to four (all correct answers). Therefore, if a student was correct about the design and descriptive statistics but incorrectly identified the inferential statistics and how to graph the data, the student received a total score of two. Mean accuracy for the group that used the Design-Statistics Finder for the first problem was 3.6 $(S D=9)$ for Problem One and $1.4(S D=1.2)$ for Problem Two. Mean accuracy for the group that solved the first problem without assistance was $1.8(S D=1.3)$ for the first problem and 1.7 $(S D=.9)$ for the second problem. A repeated measures ANOVA was conducted, specifying order as a between-subjects variable. The results show significant effects of order $[F(1,24)=5.17, p<$ $.05]$ and problem $[F(1,24)=15.35, p<.001]$ and a significant interaction between order and problem $[F(1,24)=$ $13.46, p<.001]$. The students who solved the first problem with the tutorial were more accurate than the students who did not use the tutorial. In addition, not using the tutorial on the second problem after using it on the first problem resulted in a significant decrease in accuracy. However, using the tutorial on the second problem after not using it on the first problem produced no change in accuracy. Thus, the results suggest that incorrectly solving an initial problem produces similarly poor performance on additional problems, even when tutorial help is provided. Unfortunately, using the tutorial to correctly solve the first problem does not result in transfer of learning to the second problem.

Apart from the quantitative findings, qualitative data suggest that the tutorial was beneficial for developing a strategy to solve the problem. The students who used the tutorial on the first problem were fairly accurate in their decisions about the design and statistics for the study. When they did not use the tutorial to answer the second problem, their accuracy significantly decreased. However, the type of responses they provided followed the organization of the tutorial. The students who did not use the tutorial to solve the first problem displayed no clear pattern of problem solving. Likewise, when they used the computer on the second problem. they were often unable to correctly structure the problem. Perhaps extensive practice with the on-line tutorial would help students develop an algorithm for solving design- and statisticsrelated problems. This would allow students to correctly identify the design of a study and the appropriate statistical analysis, once the variables have been correctly iden- 
tified. However, failure to correctly structure the problem appears to hinder performance even when additional resources are provided (Simon \& Hayes, 1976).

It is also interesting to note that the students who used the on-line tutorial for the first problem spent approximately five times the amount of time solving the problem than did the group who did not use the tutorial on the first problem. This finding is interesting because these students were the most accurate and research has shown that experts spend more time defining and elaborating a problem than do novices (Voss \& Post, 1988).

\section{Confidence in Problem Solving}

A similar pattern of results was found for problemsolving confidence. Mean confidence for the group that used the Design-Statistics Finder for the first problem was $12.4(S D=3.9)$ for Problem One and $8.7(S D=4.8)$ for Problem Two. Mean confidence for the group that solved the first problem without assistance was 10.4 $(S D=3.3)$ for the first problem and $9.3(S D=3.6)$ for the second problem. A repeated measures ANOVA was conducted, specifying order as a between-subjects variable. The results show a significant main effect of problem $[F(1,24)=24.11, p<.001]$ and a significant interaction between order and problem $[F(1,24)=6.85, p<$ $.015]$. There was no effect of order. The students who solved the first problem with the tutorial were more confident than students who did not use the tutorial. In addition, not using the tutorial on the second problem after using it on the first problem significantly decreased confidence. However, using the tutorial on the second problem after not using it on the first problem produced no change in confidence.

Qualitative data again provide support for the idea that the tutorial helps to properly organize the problems. The students who used the tutorial first were more accurate and more confident than those who did not. However, several of the students who solved the first problem without the tutorial reported that they were unsure about how to solve the problem and that this uncertainty remained even when they used the tutorial to guide them through the second problem. Thus, incorrectly solving the first problem not only affects later accuracy but later confidence as well.

\section{Ease of Problem Solving}

The results for problem-solving ease were similar to those for problem-solving confidence. Mean ease for the group that used the Design-Statistics Finder for the first problem was $13.7(S D=3.3)$ for Problem One and 7.9 $(S D=4.5)$ for Problem Two. Mean accuracy for the group that solved the first problem without assistance was $10.1(S D=2.6)$ for the first problem and 9.8 $(S D=3.4)$ for the second problem. A repeated measures ANOVA was conducted, specifying order as a between-subjects variable. The results show a significant main effect of problem $[F(1,24)=16.84, p<.001]$ and a significant interaction between order and problem $[F(1,24)=13.13$, $p<.001]$. There was no effect of order. As above, the stu- dents thought the first problem was easier to solve when using the tutorial. In addition, not using the tutorial on the second problem after using it on the first problem significantly increased perceived problem difficulty. However, using the tutorial on the second problem after not using it on the first problem produced no change in difficulty.

\section{Practice and Problem Solving}

A second study was conducted in order to further examine the affect of practice with the tutorial on problemsolving ability. Nine students from a statistics class and 14 from a research methods course were randomly assigned to the tutorial group, whereas 6 statistics and 12 research methods students were randomly assigned to the textbook group. Each group received eight practice problems. The problems described both true experiments and correlational studies and within- and between-subjects designs, and they required a range of statistical tests covered in introductory statistics courses (e.g., $t$ tests, ANOVAs, correlation, chi-square). The students were required to identify the independent and dependent variables, the design of the study, and the appropriate statistical analysis. The tutorial group used the Design-Statistics Finder to solve the problems in a computer lab on campus. The textbook group was allowed to use a number of statistics and research methods texts to answer the problems. This group completed the items in a research lab. Two days after the practice problems were completed, both groups met in a large classroom and were administered a second set of eight problems in which they were to identify the variables, design, and analysis for each problem, as they did during practice. Problems in the second set were similar to those in the first. Both groups solved the problems without the use of texts or the tutorial. The subjects were given $50 \mathrm{~min}$ to solve the problems. Scores were obtained by dividing the total number of correct responses by the total possible number. Since 4 responses were requested for each problem, there were 32 possible responses. The tutorial group $(M=69.7$, $S D=9.16$ ) scored significantly higher $[t(39)=2.97, p<$ $.005]$ on the test problems than did the textbook group $(M=58.71, S D=14.4)$. Thus, practice with the tutorial appears to significantly enhance students' ability to solve design- and statistics-related problems.

\section{SUMMARY}

The Design-Statistics Finder is a Web-based tutorial based on the Koch and Gobell (1999) decision charts. Because of the flexibility of the Web, the tutorial includes more information than can reasonably be included in a paper version of the decision charts. Students who initially use the Design-Statistics Finder tutorial to solve a problem tend to be more accurate and more confident in their decisions and find the decisions easier to make than do students who do not use the tutorial. In addition, students who do not use the tutorial to solve an initial problem tend to solve a second problem in the same in- 
effective manner. Their confidence in problem solving and the perceived difficulty of the problem also do not change. Thus, the tutorial seems to help students correctly structure and solve research problems, whereas not using the tutorial appears to develop an ineffective problemsolving strategy. The second study further shows that extended practice with the tutorial produces more effective problem solving on subsequent problems.

The results highlight an important point for teaching statistics and research methods. Students who initially experience difficulties solving design and statistics problems will typically experience difficulty in later problems. They will be less accurate and less confident and will find the task to be more difficult than do students who experience early success. This tendency may be most frequently observed by those teaching statistics courses in which students experience math or statistics anxiety. Therefore, it is important for instructors to incorporate learning experiences in which students can achieve success early in the learning process. Tools such as the Design-Statistics Finder tutorial can be used as one avenue to accomplish this goal.

\section{REFERENCES}

BEAR, G. (1995). Computationally intensive methods warrant reconsideration of pedagogy in statistics. Behavior Research Methods, Instruments, \& Computers, 27, 144-147.

CozBy, P. C. (1997a). Methods in behavioral research (6th ed.). Mountain View, CA: Mayfield.

CozBy, P. C. (1997b). Methods in behavioral research [On line]. Available URL: http://methods. fullerton.edu

DE LEEUW, J. (1997). Statistics: The study of variability [On line]. Available URL: http://www.stat.ucla.edu/textbook

DUNN, D. S. (1996). Collaborative writing in a statistics and research methods course. Teaching of Psychology, 23, 38-40.

ElMES, D. G. (1995). Instructor's manual with test bank to accompany research methods in psychology (5th ed.). New York: West.

EsTES, W. K. (1997). On the communication of information by displays of standard errors and confidence intervals. Psychonomic Bulletin \& Review, 4, 330-341.

KoCH, C., \& GOBELl, J. (1997). Design-statistics finder [On line]. Available URL: http://www.georgefox.edu/people/faculty/ckoch/page _ l.html KOCH, C., \& GoBeLL, J. (1999). Integrating statistics and graphing data with theory and research design. Manuscript submitted for publication.
LANE, D. M. (1997). Hyperstat online [On line]. Available URL: http:// www.ruf.rice.edu/ lane/hyperstat/contents.html

LoFTUS, G. R. (1993). A picture is worth a thousand $p$ values: On the irrelevance of hypothesis testing in the microcomputer age. Behavior Research Methods, Instruments, \& Computers, 25, 250-256.

Loftus, G. R. (1996). Psychology will be a much better science when we change the way we analyze data. Current Directions in Psychological Research, 5, 161-171.

LUTSKY, N. (1986). Undergraduate research experience through the analysis of data sets in psychology courses. Teaching of Psychology, 13, 119-122.

Pedhazur, E. J., \& Schmelkin, L. P. (1991). Measurement, design, and analysis: An integrated approach. Hillsdale, $\mathrm{NJ}$ : Erlbaum.

Rosnow, R. L., \& Rosnow, M. (1992). Writing papers in psychology: A student guide (2nd ed.). Belmont, CA: Wadsworth.

Shaughnessy, J. J., \& ZechmeIsTer, E. B. (1997). Research methods in psychology (4th ed.). New York: McGraw-Hill.

Simon, H. A., \& Hayes, J. R. (1976). The understanding process: Problem isomorphs. Cognitive Psychology, 8, 165-190.

THOMPSON, W. B. (1994). Making data analysis realistic: Incorporating research into statistics courses. Teaching of Psychology, 21, 41-43.

TRоснім, W. (1997a). Bill Trochim's center for social research methods [On line]. Available URL: http://trochim.human.cornell.edu

TrochIM, W. (1997b). Research methods tutorials [On line]. Available URL: http:/trochim.human.cornell.edu/tutorial/TUTORIAL.htm

Velleman, P. F., \& Wilkinson, L. (1993). Nominal, ordinal, interval, and ratio typologies are misleading. American Statistician, 47, 65-72.

Voss, J., \& Post, T. (1988). On the solving of ill-structured problems. In M. Chi, R. Glaser, \& M. Farr (Eds.), The nature of expertise (pp. 261285). Hillsdale, NJ: Erlbaum.

\section{NOTE}

1. We would like to emphasize three points made throughout the paper. First, this tutorial is currently designed to supplement class material traditionally used in either statistics or research methods courses. Although the flexibility of hypertext and the Web provide numerous possibilities for expansion and use of this tutorial, it should not be used as the sole source of statistical or research methods information at this time. Second. the tutorial should be used as one in a number of tools for teaching statistics and research methods. Finally, the tutorial is primarily designed for students taking their first course in statistics and/or research methods. Therefore, advanced or uncommon statistical procedures and complex research designs may not be adequately represented in the tutorial, in order to help students begin to develop statistical and research methods problem-solving strategies without being confused by a large number of statistical tests or case-specific designs.

(Manuscript received November 11, 1997; revision accepted for publication August 19, 1998.) 